\title{
Localized modes in dense repulsive and attractive Bose-Einstein condensates with spin-orbit and Rabi couplings
}

\author{
Luca Salasnich ${ }^{1}$ and Boris A. Malomed ${ }^{2}$ \\ ${ }^{1}$ Dipartimento di Fisica e Astronomia "Galileo Galilei" and CNISM, \\ Università di Padova, Via Marzolo 8, 35131 Padova, Italy \\ ${ }^{2}$ Department of Physical Electronics, School of Electrical Engineering, \\ Faculty of Engineering, Tel Aviv University, Tel Aviv 69978, Israel
}

\begin{abstract}
We consider a binary Bose-Einstein condensate with linear and nonlinear interactions between its components, which emulate the spinor system with spin-orbit (SO) and Rabi couplings. For a relatively dense condensate, $1 \mathrm{D}$ coupled equations with the nonpolynomial nonlinearity of both repulsive and attractive signs are derived from the 3D Gross-Pitaevskii equations. Profiles of modes confined in an external potential under the action of the self-repulsion, and self-trapped solitons in the case of the self-attraction, are found in a numerical form and by means of analytical approximations. In the former case, the interplay of the SO and Rabi couplings with the repulsive nonlinearity strongly distorts shapes of the trapped modes, adding conspicuous side lobes to them. In the case of the attractive nonlinearity, the most essential result is reduction of the collapse threshold under the action of the SO and Rabi couplings.
\end{abstract}

PACS numbers: $03.75 . \mathrm{Ss}, 03.75 . \mathrm{Hh}, 64.75 .+\mathrm{g}$

\section{INTRODUCTION}

The recently proposed emulation of the spin-orbit (SO) coupling in condensed matter by means of similar but weak (i.e., theoretically tractable and experimentally controllable) interactions in binary Bose-Einstein condensates (BECs) 1] has drawn much attention, in the spirit of using dilute Bose gases as quantum simulators [2]. In this context, BEC composed as mixtures of different atomic states may represent pseudo-spin systems, with the multi-component mean-field wave functions emulating the spinor order parameter. In experiments, the artificial SO coupling has been implemented in both bosonic [3, 4] and fermionic [5, 6] atomic gases, by means of counterpropagating laser beams which couple two internal hyperfine states of the atom by a stimulated twophoton Raman transition. In this connection, it is relevant to mention that the true $\mathrm{SO}$ coupling (rather than the emulated one) is possible too in BEC composed of spinor bosons [7] and in magnon condensates [8].

The single-particle SO Hamiltonian which can be implemented in the BEC is

$$
\hat{h}_{\mathrm{sp}}=\left[\frac{\hat{\mathbf{p}}^{2}}{2 m}+U(\mathbf{r})\right]+\frac{\hbar \Omega}{2} \sigma_{x}-\frac{k_{L}}{m} \hat{p}_{x} \sigma_{z}
$$

where $\hat{\mathbf{p}}=-i \hbar\left(\partial_{x}, \partial_{y}, \partial_{z}\right)$ is the momentum operator, $U(\mathbf{r})$ is a trapping potential, $k_{L}$ is the recoil wavenumber induced by the interaction with the laser beams, $\Omega$ is the frequency of the Raman coupling, which is responsible for the Rabi mixing between the two states, and $\sigma_{x, z}$ are the Pauli matrices. Recently, considerable attention has been drawn to models combining the SO coupling, which is a linear feature, and mean-field nonlinearities, which are induced, as usual [9], by inter-atomic collisions. Various dynamical effects have been investigated in the framework of such nonlinear systems, including the self-trapping and formation of solitons [11, 12], vortical patterns [13], the interplay of the SO coupling and dipole-dipole interactions [14], the modulational instability of uniform states in SO-coupled BEC [15], etc.

The objective of the present work is to present an effective 1D model for the SO- and Rabi-coupled relatively dense binary $\mathrm{BEC}$, and investigate basic properties if trapped modes in the framework of the model. The $d i$ mension reduction, i.e., derivation of the $1 \mathrm{D}$ system from the underlying set of the 3D Gross-Pitaevskii equations (GPEs) with cubic terms, leads to nonpolynomial nonlinearity, as was previously demonstrated in the context of single- [16] and binary [18] condensates with the attractive nonlinearity, as well as for the repulsive nonlinearity [19]. In the former case, the corresponding nonpolynomial nonlinear Schrödinger equations (NPSEs) generate solitons, as the usual 1D GPEs with the postulated cubic nonlinearity. However, on the contrary to the 1D cubic equations, the NPSEs predict the onset of collapse in the effectively 1D solitons at a critical strength of the selfattraction [16]. This feature makes the 1D solitons similar to 3D solitons, which are also subject to the collapse above a critical value of the number of atoms, depending on parameters of the BEC-trapping potential. Detailed numerical investigations have demonstrated that the near-collapse dynamics of 3D solitons (although not the collapse itself) is quite accurately approximated by their 1D counterparts in the framework of the NPSE [17].

In this work, we derive a system of two NPSEs coupled by linear and nonlinear terms, which include new terms accounting for the emulated SO interaction. The derivation is performed for both the repulsive and attractive signs of the inter-atomic interactions, and is followed by the analysis of trapped modes, for both signs of the nonlinearity. In the case of the self-repulsion, the trapping is imposed by an axial harmonic-oscillator (HO) potential, while the attractive condensates self-traps into solitons. 
The analysis concentrates on new features of the externally trapped and self-trapped modes in the presence of the SO-emulating interactions.

The rest of the paper is organized in the following way. The derivation of the NPSE system for the binary condensate with the $\mathrm{SO}$ and Rabi interactions between the components is reported in Section II. This section also includes analytical approximations which help to understand characteristic features of the trapped modes in the SO-coupled dense condensates. Basic numerical results are reported in Section III. In both cases of the selfrepulsion and self-attraction, the nonpolynomial nonlinearity strongly affects shapes of the trapped modes and solitons. The most essential result is a reduction of the collapse threshold for the solitons under the action of the $\mathrm{SO}$ and Rabi interactions. The paper is concluded by Section IV.

\section{SPIN-ORBIT COUPLED NONPOLYNOMIAL SCHRÖDINGER EQUATIONS}

\section{A. Derivation of the model}

Our starting point is the $3 \mathrm{D}$ version of the $\mathrm{SO}$ system which was recently introduced in the form of 1D GPEs with the cubic nonlinearity [11]. In fact, the 1D equations postulated in Ref. [11] are essentially the same as those derived earlier in the context of nonlinear fiber optics, for two polarizations of light co-propagating in a twisted birefringent fiber [20]). A different version of the 1D SO Hamiltonian was adopted in another recent paper [12]. If one takes into account the nonlinearity induced by atomic collisions, the present model and the one studied in Ref. 12] can be transformed into each other, provided that the strength of the nonlinear terms is characterized by the single $s$-wave scattering length.

The SO- and Rabi-coupled binary BEC, confined in the plane of $(y, z)$ by a tight $\mathrm{HO}$ potential with trapping frequency $\omega_{\perp}$, and in the $x$ direction by a generic loose potential $V(x)$, is modeled by the system of 3D GPEs for macroscopic wave functions $\psi_{k}$ of the two atomic states $(k=1,2)$ :

$$
\begin{aligned}
i \partial_{t} \psi_{k} & =\left[-\frac{1}{2} \nabla^{2}+V(x)+\frac{1}{2}\left(y^{2}+z^{2}\right)+(-1)^{k-1} i \gamma \partial_{x}\right. \\
& \left.+2 \pi g_{k}\left|\psi_{k}\right|^{2}+2 \pi g_{12}\left|\psi_{3-k}\right|^{2}\right] \psi_{k}+\Gamma \psi_{3-k},
\end{aligned}
$$

where the lengths, time, and energy are measured in units of $a_{\perp}=\sqrt{\hbar /\left(m \omega_{\perp}\right)}, \omega_{\perp}^{-1}$, and $\hbar \omega_{\perp}$, respectively. Here $g_{k} \equiv 2 a_{k} / a_{\perp}, g_{12} \equiv 2 a_{12} / a_{\perp}$ are strengths of the intra- and inter-species interactions, with the respective scattering lengths $a_{k}$ and $a_{12}$, while $\gamma \equiv k_{L} a_{\perp}$ and $\Gamma \equiv \Omega /\left(2 \omega_{\perp}\right)$ are dimensionless strengths of the $\mathrm{SO}$ and Rabi couplings, respectively [recall that $k_{L}$ is the recoil wavenumber in Hamiltonian (1)]. The timedependent number of atoms in the $k$-th state is $N_{k}(t)=$ $\iiint d x d y d z\left|\psi_{k}(x, y, z, t)\right|^{2}$, the constant total number of atoms being $N=N_{1}(t)+N_{2}(t)$.

To reduce the dimension from $3 \mathrm{D}$ to $1 \mathrm{D}$, we adopt the usual factorized ansatz for the wave functions which are tightly trapped in the transverse plane, $(y, z)$, and weakly confined in the axial direction, $x$ [16, 18]:

$$
\psi_{k}(x, y, z, t)=\frac{1}{\sqrt{\pi} \eta_{k}(x, t)} \exp \left\{-\frac{y^{2}+z^{2}}{2 \eta_{k}^{2}(x, t)}\right\} f_{k}(x, t),
$$

where $\eta_{k}(x, t)$ and $f_{k}(x, t)$ are the transverse widths and axial wave functions, respectively, the latter normalized by conditions

$$
\int_{-\infty}^{+\infty}\left|f_{k}(x, t)\right|^{2} d x=N_{k}(t)
$$

which are compatible with Eq. (3). The conserved total number of atoms is $N=N_{1}+N_{2}$.

Inserting ansatz (3) into the Lagrangian density which produces Eqs. (2), performing the integration in the transverse plane, and neglecting, as usual, derivatives of $\eta_{k}(x, t)$, one can derive the corresponding effective Lagrangian, which then gives rise to a system of four variational equations:

$$
\begin{gathered}
i \partial_{t} f_{k}=\left[-\frac{1}{2} \partial_{x}^{2}+V(x)+(-1)^{k-1} i \gamma \partial_{x}+\frac{1}{2}\left(\frac{1}{\eta_{k}^{2}}+\eta_{k}^{2}\right)+\right. \\
\left.\frac{g_{k}}{\eta_{k}^{2}}\left|f_{k}\right|^{2}+2 \frac{g_{12}}{\left(\eta_{1}^{2}+\eta_{2}^{2}\right)}\left|f_{3-k}\right|^{2}\right] f_{k}+2 \Gamma \frac{\eta_{1} \eta_{2}}{\left(\eta_{1}^{2}+\eta_{2}^{2}\right)} f_{3-k} \\
\eta_{k}^{4}=1+g_{k}\left|f_{k}\right|^{2}+4 g_{12}\left|f_{3-k}\right|^{2} \frac{\eta_{k}^{4}}{\left(\eta_{1}^{2}+\eta_{2}^{2}\right)^{2}} \\
+2(-1)^{k-1} \Gamma \frac{\left(f_{1}^{*} f_{2}+f_{2}^{*} f_{1}\right)}{\left|f_{1}\right|^{2}} \frac{\eta_{k}^{3} \eta_{3-k}\left(\eta_{1}^{2}-\eta_{2}^{2}\right)}{\left(\eta_{1}^{2}+\eta_{2}^{2}\right)^{2}} .
\end{gathered}
$$

In most cases, a reasonable assumption is that strengths of the nonlinear interactions between different species are equal,

$$
g_{1}=g_{2}=g_{12} \equiv g
$$

21] [in fact, a more general case is considered below too, see Eqs. (13) and (15)]. In this case, algebraic equations (6) admit a simple solution, making it possible to eliminate the widths in favor of the wave functions:

$$
\eta_{1}^{4}=\eta_{2}^{4}=1+g\left(\left|f_{1}\right|^{2}+\left|f_{2}\right|^{2}\right),
$$

hence equations (5) for the two coupled axial wave functions may be cast into a closed form,

$$
\begin{gathered}
i \partial_{t} f_{k}=\left[-\frac{1}{2} \partial_{x}^{2}+V(x)+(-1)^{k-1} i \gamma \partial_{x}\right. \\
\left.+\frac{1+(3 / 2) g\left(\left|f_{1}\right|^{2}+\left|f_{2}\right|^{2}\right)}{\sqrt{1+g\left(\left|f_{1}\right|^{2}+\left|f_{2}\right|^{2}\right)}}\right] f_{k}+\Gamma f_{3-k} .
\end{gathered}
$$



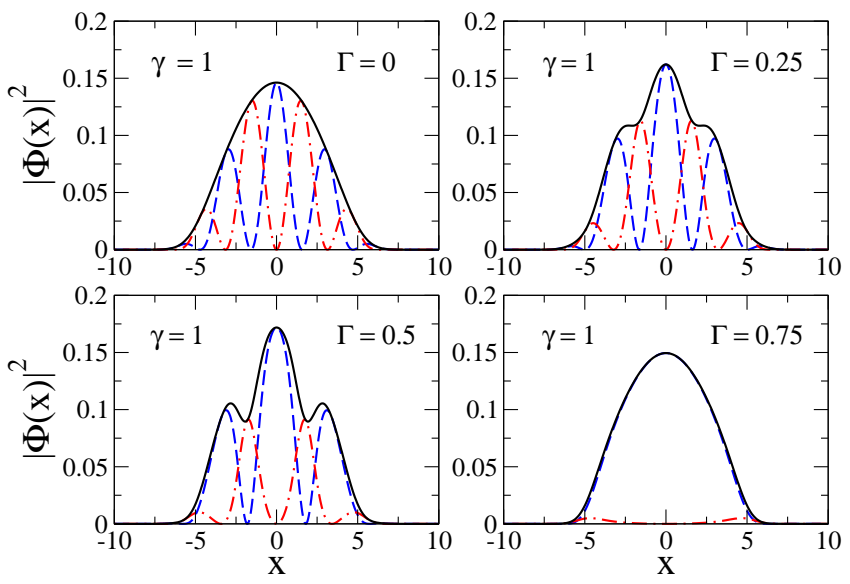

FIG. 1: (Color online). The repulsive binary BEC under axial harmonic confinement (26): The probability density for the trap anisotropy $\lambda=1 / 3$, adimensional nonlinearity strength $g N=20$, spin-orbit coupling $\gamma=1$, and four values of the adimensional Rabi coupling $\Gamma$. Here, and in similar plots displayed below, the solid line depicts $|\Phi(x)|^{2}=\Phi_{R}^{2}(x)+\Phi_{I}^{2}(x)$, while the dashed and dashed-dotted lines separately represent squared real and imaginary parts of the wave function, $\Phi_{R}^{2}(x)$ and $\Phi_{I}^{2}(x)$. Lengths are measured, here and below, in units of the transverse confinement radius, $a_{\perp}=\sqrt{\hbar /\left(m \omega_{\perp}\right)}$.

This NPSE system is a generalization of the one introduced earlier [18] for the study of vectorial solitons in two-component BECs, under the same assumption (7D) as adopted here (but without the linear coupling between the components).

Using Eq. (9), we aim to construct stationary states with chemical potential $\mu$, setting

$$
f_{k}(x, t)=\phi_{k}(x) e^{-i \mu t} .
$$

The resulting equations for stationary fields $\phi_{1,2}(x)$ are compatible with restriction

$$
\phi_{1}^{*}(x)=\phi_{2}(x),
$$

leading to the single stationary NPSE:

$\mu \Phi=-\frac{1}{2} \Phi^{\prime \prime}+V(x) \Phi+i \gamma \Phi^{\prime}+\frac{1+(3 / 2) g N|\Phi|^{2}}{\sqrt{1+g N|\Phi|^{2}}} \Phi+\Gamma \Phi^{*}$,

where we have set $\Phi(x) \equiv \sqrt{2 / N} \phi_{1}(x)=\sqrt{2 / N} \phi_{2}^{*}(x)$, so that $\int_{-\infty}^{+\infty} d x|\Phi(x)|^{2}=1$, and the prime stands for $d / d x$. Clearly, solutions of Eq. (12) are complex if $\gamma \neq$ 0 , with the symmetry which implies that their real and imaginary parts, $\Phi_{R}(x)$ and $\Phi_{I}(x)$, are, respectively, even and odd functions, provided that potential $V(x)$ is even (or is absent).

In fact, being interested in stationary solutions subject to constraint (11), we can also consider a case more general than the one singled out by Eq. (77) (the full symmetry of the nonlinear interactions). This generalization is essential because the case of the full symmetry is critical, in some respects [21]. Assuming $\left|f_{1}\right|^{2}=\left|f_{2}\right|^{2} \equiv|f|^{2}$ and

$$
g_{1}=g_{2} \equiv g \neq g_{12},
$$

one can again obtain a simple solution for algebraic equations (6):

$$
\eta_{1}^{4}=\eta_{2}^{4}=1+\left(g+g_{12}\right)|f|^{2},
$$

the substitution of which into Eqs. (5), (10) and (11) leads to the stationary equation tantamount to Eq. (12), with $g$ replaced, according to Eqs. (13) and (14), by

$$
g_{\mathrm{eff}} \equiv(1 / 2)\left(g+g_{12}\right) .
$$

All the stationary solutions obtained from Eq. (12) pertain to the solutions subject to condition (11), if $g$ is replaced by $g_{\text {eff }}$ in the case when relation (13) holds. This equivalence does not apply to asymmetric solutions, which do not obey relation (11). Strictly speaking, the equivalence does not pertain either to the study of the dynamical solutions of the solutions, which must be carried out withing the framework of full equations (9), and, in particular, should include perturbations which may break relation (11).

Finally, we note that, in the weakly nonlinear regime, $g N|\Phi|^{2} \ll 1$, the nonpolynomial term in Eq. (12) may be expanded and reduced to the cubic approximation:

$$
(\mu-1) \Phi=-\frac{1}{2} \Phi^{\prime \prime}+V(x)+i \gamma \Phi^{\prime}+g N|\Phi|^{2} \Phi+\Gamma \Phi^{*} .
$$

In the opposite limit of $g N|\Phi|^{2} \gg 1$, which may be relevant in the case of the repulsive interactions, the nonpolynomial nonlinearity reduces to a quadratic form, $\sim|\Phi| \Phi$, cf. Ref. [19].

\section{B. Analytical approximations}

In the absence of the Rabi coupling, $\Gamma=0$, the SO term can be removed from Eq. (12) by substitution

$$
\Phi(x) \equiv \Phi_{0}(x) e^{i \gamma x},
$$

the resulting equation for $\Phi_{0}(x)$ being tantamount to the stationary version of the usual NPSE [16], with a shifted chemical potential,

$$
\tilde{\mu} \equiv \mu+\gamma^{2} / 2 .
$$

The same substitution (17) may be used to produce an analytical result valid for small $\Gamma$ and/or large $\gamma$ : in the lowest approximation, the solution is

$$
\Phi(x) \approx \Phi_{0}(x) e^{i \gamma x}\left[1+\frac{i \Gamma}{2 \gamma^{2}} \sin (2 \gamma x)\right],
$$

where $\Phi_{0}(x)$ is, as said above, a real solution for the usual NPSE with $\Gamma=\gamma=0$ and chemical potential (18). 
Note that this approximation predicts an increase of the height of the density profile in the mode, averaged over oscillations between the real and imaginary parts of the wave function:

$$
\overline{|\Phi(x)|^{2}} \approx \Phi_{0}^{2}(x)\left(1+\Gamma^{2} / 8 \gamma^{4}\right) .
$$

In the opposite case, when $\gamma$ is small and $\Gamma$ is large, an analytical approach can be developed too. In this situation, a straightforward consideration of Eq. (12) demonstrates that, in the lowest approximation, the solution can be constructed as one with a small imaginary part:

$$
\Phi(x) \approx \Phi_{0}(x)+\frac{i \gamma}{2 \Gamma} \Phi_{0}^{\prime}(x),
$$

where $\Phi_{0}(x)$ is, as above, the solution of the usual NPSE corresponding to the given norm, $N$.

Finally, a specific approximation applies to the description of broad solitons, for which the kinetic-energy term in Eq. (9), $(1 / 2) \partial_{x}^{2} f_{k}$, may be neglected in comparison with the SO coupling, $(-1)^{k-1} i \gamma \partial_{x} f_{k}$. Broad solitons may be naturally assumed to have a small amplitude, hence the accordingly expanded system of equations (9) is approximated by

$$
\begin{gathered}
i \partial_{t} F_{k}=\left[V(x)+(-1)^{k-1} i \gamma \partial_{x}\right. \\
\left.+g\left(\left|F_{1}\right|^{2}+\left|F_{2}\right|^{2}\right)\right] F_{k}+\Gamma f_{3-k},
\end{gathered}
$$

where $F_{k} \equiv f_{k} \exp (i t)$. In the absence of the axial potential $(V=0)$, the self-attractive nonlinearity $(g<0)$ gives rise to well-known exact solutions of Eq. (22) in the form of gap solitons, which may be moving ones, with velocity $c$ [22, 23], namely

$$
\begin{aligned}
F_{1}= & \frac{1}{\gamma} \sqrt{\Gamma \frac{\gamma-c}{2|g|}}\left(\gamma^{2}-c^{2}\right)^{1 / 4} W^{*}(X) . \\
& \exp [i \phi(X)-i T \cos \theta], \\
F_{2}= & \frac{1}{\gamma} \sqrt{\Gamma \frac{\gamma+c}{2|g|}}\left(\gamma^{2}-c^{2}\right)^{1 / 4} W(X) . \\
& \exp [i \phi(X)-i T \cos \theta],
\end{aligned}
$$

where

$$
\begin{aligned}
X & =\Gamma\left(\gamma^{2}-c^{2}\right)^{-1 / 2}(x-c t), \\
T & =\left(\gamma^{2}-c^{2}\right)^{-1 / 2}(\Gamma / \gamma)\left(\gamma^{2} t-c x\right), \\
\phi(X) & =(2 c / \gamma) \tan ^{-1}\{\tanh [(\sin \theta) X] \tan (\theta / 2)\}, \\
W(X) & =(\sin \theta) \operatorname{sech}[(\sin \theta) X-i(\theta / 2)],
\end{aligned}
$$

and the parameter $\theta$ is determined by the normalization condition, $N=N_{1}+N_{2}$ [see Eq.(4)],

$$
\theta=(|g| \gamma / 2)\left(\gamma^{2}-c^{2}\right)^{-1} N .
$$

The solitons exist with velocities $c^{2}<\gamma^{2}$, and with $\theta<\pi$, which imposes a limitation on the number of atoms in
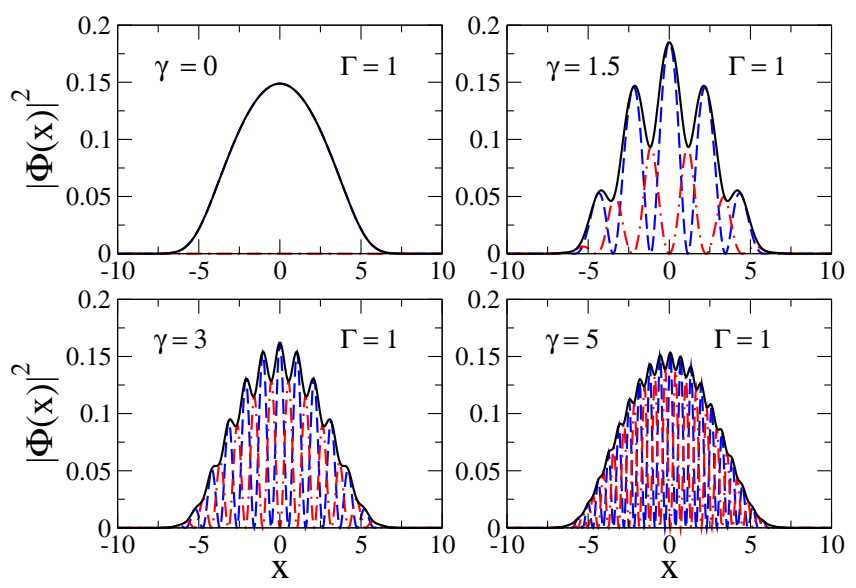

FIG. 2: (Color online). The same as in Fig. 1) but for a fixed Rabi coupling, $\Gamma=1$, and four different values of the spin-orbit coupling, $\gamma$.

the soliton of this type, according to Eq. (25). In fact, the solitons are stable, approximately, in the interval of $\theta<\pi / 2$ [24], which makes the limitation on $N$ more restrictive.

The broad gaps solitons can be also found in the presence of the axial trapping potential, $V(x)$, in Eq. (22). Indeed, for the broad soliton it may be approximated by $V(x) \approx-V_{0} \delta(x)$ with $V_{0}>0$. Quiescent gap solitons (with $c=0$ ) can be found in an exact analytical form in this case too 25].

The gap solitons exist as well in the case of the selfrepulsion, i.e., $g>0$. The respective solutions are obtained from Eqs. (23) and (24), substituting $\left\{F_{1}, F_{2}\right\}$ by $\left\{F_{1}^{*},-F_{2}^{*}\right\}$.

Lastly, the underlying condition for neglecting the second derivatives in Eq. (9) and reducing it to Eq. (22) takes the form of $N \ll(\Gamma|g|)^{-1}\left(\gamma^{2}-c^{2}\right)^{3 / 2}$. Of course, the gap solitons do not exist in the rigorous sense, as the neglected second derivatives close the spectral gap, giving rise to a slow decay of the solitons into radiation waves, cf. Ref. [26].

\section{NUMERICAL RESULTS}

\section{A. The self-repulsive spin-orbit-coupled BEC under the harmonic-oscillator axial confinement}

We start the numerical analysis by looking for trapped modes supported by Eq. (12) with $g>0$ (the repulsive nonlinearity) in the presence of the $\mathrm{HO}$ axial potential with frequency $\omega_{x}$,

$$
V(x)=\left(\lambda^{2} / 2\right) x^{2},
$$

where $\lambda \equiv \omega_{x} / \omega_{\perp}$ is the anisotropy of the HO confinement. Equation (12) was solved by dint of the imaginarytime method, implemented with the help of the finite- 
difference predictor-corrector Crank-Nicolson algorithm [27]. In Fig. 1 the numerical results are reported for the HO potential (26) with $\lambda=1 / 3$, adimensional nonlinearity strength $g N=20$, SO coupling $\gamma=1$, and four different values of Rabi coupling $\Gamma$. The density profile of the trapped modes, $|\Phi(x)|^{2}$, is plotted as a function of axial coordinate $x$ (solid lines). For the completeness of the depiction of the complex wave functions, we also display $\Phi_{R}^{2}(x)$ and $\Phi_{I}^{2}(x)$ (dashed and dot-dashed lines, respectively). As expected, for $\Gamma=0$ (the left upper panel of Fig. 1) density $|\Phi(x)|^{2}$ for $\gamma=1$ is the same as for $\gamma=0$. In this case, a finite value of $\gamma$ implies that $\Phi(x)$ has oscillatory real and imaginary components $\Phi_{R}(x)$ and $\Phi_{I}(x)$. The increase of $\Gamma$ at fixed $\gamma(\gamma=1$ in Fig. 1) leads to the decrease of the imaginary part, the corresponding density profile, $|\Phi(x)|^{2}$, displaying several local maxima (see the right top and left bottom panels in Fig. 1). Finally, when $\Gamma$ is sufficiently large, the imaginary component of the density, $\Phi_{I}^{2}(x)$, becomes very small, in accordance with analytical approximation (21), the probability density $|\Phi(x)|^{2}$ being nearly identical to that at $\Gamma=0$, as can be seen from the comparison of the right bottom and left top panels in Fig. 1).

It is also relevant to analyze the density profile at fixed $\Gamma$ for different values of the SO coupling $\gamma$. To this end, in Fig. 2 we display the numerical results for $\lambda=1 / 3$, $g N=20, \Gamma=1$, and four values of $\gamma$. At $\gamma=0$ (the left upper panel of Fig. 2), $\Phi(x)$ is real and the density profile is smooth. As shown in Fig. 2, for $\gamma \neq 0$ solution $\Phi(x)$ is complex, and the respective density $|\Phi(x)|^{2}$ displays several local maxima, whose number increases with $\gamma$. At large values of $\gamma$, we observe many small-amplitude local variations of $|\Phi(x)|^{2}$, which makes the averaged density profile close to that at $\gamma=0$. This regime may be explained by analytical approximation (19).

To summarize, the numerical results demonstrate that the SO and Rabi couplings produce the most prominent effect on the density profile of the trapped modes in the self-repulsive binary BEC, in the form od conspicuous side lobes, in the case when the constant accounting for both couplings, $\gamma$ and $\Gamma$, take moderate values, neither very small nor too large. It is also relevant to stress that Figs. [1] and 2 demonstrate values $g N|\Phi(x=0)|^{2} \simeq 3$, at peak-density points. This implies, according to Eq. (12), that the nonpolynomiality of the effective nonlinearity is quite essential.

\section{B. Bright solitons in the self-attractive spin-orbit-coupled BEC}

It is well known that the attractive intrinsic nonlinearity $(g<0)$ supports self-trapped matter-wave solitons in the effectively 1D BEC, even in the absence of the axial confinement $[V(x)=0]$ [28]. Here we aim to investigate effects of the SO and Rabi coupling on the bright solitons by solving Eqs. (9) and (12) with $g<0$, in the absence of the axial trapping potential $[V(x)=0]$. The objective

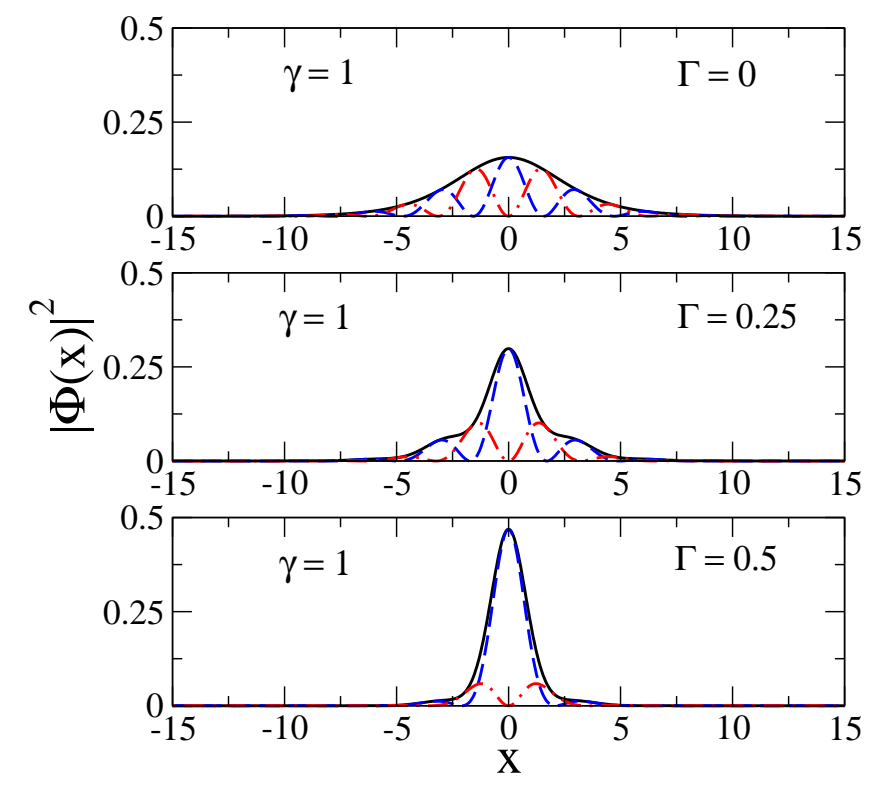

FIG. 3: (Color online). Density profiles of the solitons, in the case of the self-attractive binary BEC without the axial potential, $V=0$. Here, the nonlinearity strength is $g N=$ -0.6 , spin-orbit coupling is $\gamma=1$, and values of the Rabi coupling, $\Gamma$, are indicated in the panels.

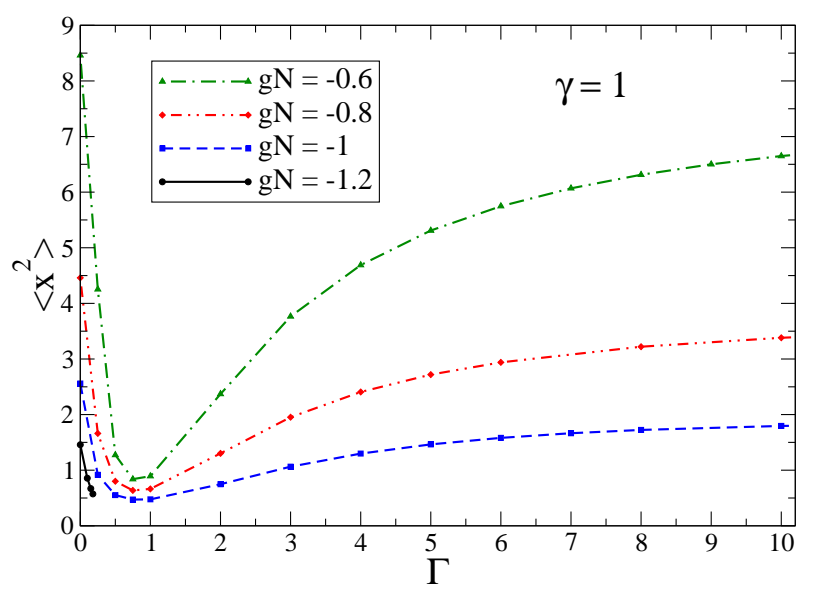

FIG. 4: (Color online). The axial squared width $\left\langle x^{2}\right\rangle$ of the solitons, defined as per Eq. (27), versus the Rabi coupling, $\Gamma$. Here the spin-orbit coupling is $\gamma=1$, and four values of the nonlinearity strength, $g N$, are indicated in the box (the curve for $g N=-1.2$ is very short, terminating because of the onset of the collapse).

is to construct true solitons, unlike the approximate gap solitons (23).

For $\gamma=0$ and/or $\Gamma=0$, Eqs. (9) and (12) with $V(x)=0$ predict that metastable solitons exist only for $-4 / 3<g N<0$. Indeed, at $g N<-4 / 3$ the $1 \mathrm{D}$ NPSE gives rise to the collapse of the condensate, which sets in locally at a point where the total density of the condensate attains the critical value, $\left|f_{1}\right|^{2}+\left|f_{2}\right|^{2}=-1 / g[$ or 
$|\Phi|^{2}=-(g N)^{-1}$, in terms of Eq. [12)]. This prediction is known to be in very good agreement with the full 3D calculations [16, 17].

Obviously interesting issues are a change of the shape of the solitons, and a shift of the collapse threshold under the action of the SO and Rabi couplings. In Fig. 3 we plot the density profile $|\Phi(x)|^{2}$ (solid line) for the bright solitons, obtained from the numerical solution of Eq. (12), for $g N=-0.6, \gamma=1$, and three values of $\Gamma$. A noteworthy feature is the compression of the soliton's density profile and increase of its height with the increase of $\Gamma$. This trend can be explained by the abovementioned perturbative result, given by Eq. (20).

At a certain finite value of $\Gamma$, the bright soliton reaches its smallest axial width and largest peak density, $|\Phi(x=0)|^{2}$, while the imaginary component (the dashed-dotted line) of $\Phi(x)$ becomes small, see the lower panel of Fig. 3. With the further increase of $\Gamma$, the imaginary part of the wave function vanishes, in accordance with Eq. (21), while the axial width approaches a finite asymptotic value, corresponding to the real solution with given norm $N$. This trend is displayed in Fig. 固 which shows the average soliton's width,

$$
\left\langle x^{2}\right\rangle \equiv N^{-1} \int_{-\infty}^{+\infty}|\Phi(x)|^{2} d x
$$

versus $\Gamma$. The four curves with symbols of Fig. 4 correspond to different values of the adimensional nonlinearity strength $g N$. Note that for $g N=-1.2$ (the solid line with filled circles) the collapse of the binary BEC happens at $\Gamma \approx 0.19$, therefore this line is aborted in Fig. 4. Thus, at fixed values of the SO coupling $(\gamma=1$ in Fig. 4) a finite Raman coupling $\Gamma$ corresponds to the lowest collapse threshold.

The results are summarized in Fig. 5, which shows the collapse threshold in the parameter space $(\gamma, \Gamma,|g| N)$ for the self-attractive binary BEC in the absence of the axial confinement. The collapse points are obtained from numerical solutions of Eq. (12), as those beyond which no solution can be found (we here do not study dynamics of the collapsing states). We stress that, in accordance with Fig. (4) the collapse is first attained at a finite value of $\Gamma$, close to the point of the minimum of the soliton's width,

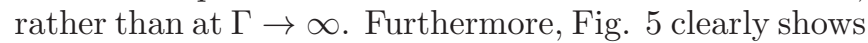
that the SO or Rabi coupling, acting in isolation, do not affect the collapse threshold at all, while the strongest reduction of the collapse threshold is produced by the interplay of these two interactions added to the binary condensate (i.e., in terms of Fig. 5 , the threshold features the steepest descent, roughly, in the diagonal direction).

To illustrate the symmetry of the system, the figure includes both positive and negative values of $\gamma$ and $\Gamma$. Note that negative $\Gamma$ corresponds to the fact that, instead of Eq. (11), one could impose constraint $\phi_{1}^{*}(x)=-\phi_{2}(x)$, which gives Eq. (12) with $\Gamma$ replaced by $-\Gamma$. While, as stressed above, for $\Gamma>0$ the real and imaginary parts of the soliton's wave function, $\Phi_{R}(x)$ and $\Phi_{I}(x)$, are even and odd, respectively, they have opposite parities for $\Gamma<$

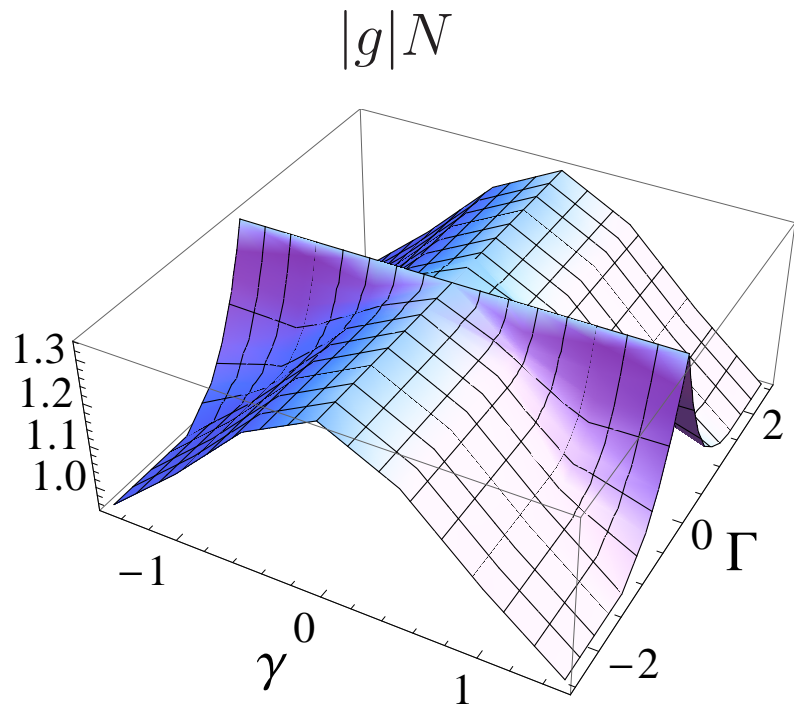

FIG. 5: (Color online). The critical value of the nonlinearity strength, corresponding to the onset of the collapse in the 1D soliton, versus the adimensional spin-orbit and Rabi couplings, $\gamma$ and $\Gamma$.

0. Further, the critical surface in Fig. [5 is symmetric with respect to the change of $\gamma \rightarrow-\gamma$ because this is tantamount to $x \rightarrow-x$.

\section{CONCLUSION}

We have derived the 1D system of coupled GrossPitaevskii equations for the relatively dense binary BEC, in which the two components are coupled by the SO (spin-orbit) and Rabi linear terms, and also by the nonlinear interactions, both selforepulsive and selfattractive. The analysis was focused on effects caused by the nonpolynomial character of the nonlinearity in the $1 \mathrm{D}$ equations derived from the system of 3D GPEs. In addition to the numerical results, analytical approximations were developed too, for the cases when the SO and Raman couplings are weak, as well as for broad trapped modes. The results demonstrate essential changes of the shape of the modes due to the nonpolynomial nonlinearity, which can be partly explained by means of the analytical approximations. In the case of the self-repulsive nonlinearity, the interplay of the SO and Rabi couplings cause a strong deformation of the shape of the trapped modes, which develop large side lobes. The most significant result is the reduction of the strength of the selfattractive nonlinearity at the collapse threshold for the effectively 1D solitons under the combined action of the SO and Rabi couplings.

An interesting possibility is to extend the present analysis for effectively $2 \mathrm{D}$ binary BEC in the presence of a tight $1 \mathrm{D}$ trapping potential acting in the transverse direction. In the absence of the linear couplings, 2D single- 
component solitons affected by the corresponding nonpolynomial nonlinearity were studied in Ref. [29]. Here too, one may expect a nontrivial lowering of the collapse threshold for the effectively 2D solitons.

\section{Acknowledgments}

LS thanks for partial support Università di Padova (Research Project "Quantum Information with Ultracold
Atoms in Optical Lattices"), Cariparo Foundation (Excellence Project "Macroscopic Quantum Properties of Ultracold Atoms under Optical Confinement"), and Ministero Istruzione Universita Ricerca (PRIN Project "Collective Quantum Phenomena: from Strongly-Correlated Systems to Quantum Simulators").
[1] C. J. Wang, C. Gao, C. M. Jian, and H. Zhai, Phys. Rev. Lett. 105, 160403 (2010); H. Zhai, Int. J. Mod. Phys. B 26, 1230001 (2012).

[2] P. Hauke, F. M. Cucchietti, L. Tagliacozzo, I. Deutsch and M. Lewenstein, Rep. Prog. Phys. 75, 082401 (2012).

[3] Y. J. Lin, K. Jimenez-Garcia, and I. B. Spielman, Nature 471, 83 (2011).

[4] J.-Y. Zhang, S.-C. Ji, Z. Chen, L. Zhang, Z.-D. Du, B. Yan, G.-S. Pan, B. Zhao, Y.-J. Deng, H. Zhai, S. Chen, and J.-W. Pan, Phys. Rev. Lett. 109, 115301 (2012).

[5] P. Wang, Z.-Q. Yu, Z. Fu, J. Miao, L. Huang, S. Chai, H. Zhai, and J. Zhang, Phys. Rev. Lett. 109, 095301 (2012).

[6] L. W. Cheuk, A. T. Sommer, Z. Hadzibabic, T. Yefsah, W. S. Bakr, and M. W. Zwierlein, Phys. Rev. Lett. 109, 095302 (2012).

[7] C. Wu, Mod. Phys. Lett. B 23, 1 (2009).

[8] Y. M. Bunkov and G. E. Volovik, J. Phys. - Cond. Matt. 22, 164210 (2010).

[9] L. Pitaevskii and S. Stringari, Bose-Einstein Condensation (Clarendon Press: Oxford, 2003).

[10] M. Merk, A. Jacob, F. E. Zimmer, P. Ohberg, and L. Santos, Phys. Rev. Lett. 104, 073603 (2010); O. Fialko, J. Brand, and U. Zuelicke, Phys. Rev. A 85, 051605 (2012); R. Liao, Z.-G. Huang, X.-M. Lin, and W.-M. Liu, ibid. 87, 043605 (2013).

[11] V. Achilleos, D. J. Frantzeskakis, P. G. Kevrekidis, and D. E. Pelinovsky, e-preprint arXiv:1211.0199.

[12] Y. Xu, Y. Zhang, and B. Wu, Phys. Rev. A 87, 013614 (2013).

[13] X.-Q. Xu and J. H. Han, Phys. Rev. Lett. 107, 200401 (2011); S. Sinha, R. Nath, and L. Santos, Phys. Rev. Lett. 107, 270401(2011); C.-F. Liu and W. M. Liu, Phys. Rev. A 86, 033602 (2012); E. Ruokokoski, J. A. Huhtamaki, and M. Mottonen, Phys. Rev. A 86, 051607 (2012); H. Sakaguchi and B. Li, Phys. Rev. A 87, 015602 (2013).

[14] Y. Deng, J. Cheng, H. Jing, C. P. Sun, and S. Yi, Phys. Rev. Lett. 108, 125301 (2012).

[15] P.-S. He, Eur. Phys. J. D 67, 48 (2013).
[16] L. Salasnich, Laser Phys. 12, 198 (2002); L. Salasnich, A. Parola, and L. Reatto, Phys. Rev. A 65, 043614 (2002).

[17] J. Cuevas, P. G. Kevrekidis, B. A. Malomed, P. Dyke, and R. G. Hulet, New J. Phys. 15, 063006 (2013).

[18] L. Salasnich and B. A. Malomed, Phys. Rev. A 74, 053610 (2006).

[19] A. Muñoz Mateo and V. Delgado, Phys. Rev. A 75, 063610 (2007); 77, 013617 (2008); Ann. Phys. (NY) 324, 709 (2009).

[20] B. A. Malomed, Phys. Rev. A 43, 410 (1991).

[21] J. Dalibard, F. Gerbier, G. Juzeliūnas, and P. Öhberg, Rev. Mod. Phys. 83, 1523 (2011).

[22] A. B. Aceves and S. Wabnitz, Phys. Lett. A 141, 37 (1989); D. N. Christodoulides and R. I. Joseph, Phys. Rev. Lett. 62, 1746 (1989).

[23] C. M. de Sterke and J. E. Sipe, in: Progr. Opt., Vol. 33, chapter 3 (ed. E. Wolf: North-Holland, Amsterdam, 1994).

[24] B. A. Malomed and R. S. Tasgal, Phys. Rev. E 49, 5787 (1994); I. V. Barashenkov, D. E. Pelinovsky, and E. V. Zemlyanaya, Phys. Rev. Lett. 80, 5117 (1998); A. De Rossi, C. Conti, and S. Trillo, ibid. 81, 85 (1998).

[25] W. C. K. Mak, B. A. Malomed, and P. L. Chu, J. Opt. Soc. Am. B 20, 725 (2003).

[26] A. R. Champneys, B. A. Malomed, and M. J. Friedman, Phys. Rev. Lett. 80, 4169 (1998).

[27] E. Cerboneschi, R. Mannella, E. Arimondo, and L. Salasnich, 249, 495 (1998); G. Mazzarella and L. Salasnich, Phys. Lett. A 373, 4434 (2009).

[28] K. E. Strecker, G. B. Partridge, A. G. Truscott, and R. G. Hulet, New J. Phys. 5, 73.1 (2003); V. A. Brazhnyi and V. V. Konotop, Mod. Phys. Lett. B 18, 627 (2004); F. Kh. Abdullaev, A. Gammal, A. M. Kamchatnov, and L. Tomio, Int. J. Mod. Phys. B 19, 3415 (2005).

[29] L. Salasnich and B. A. Malomed, Phys. Rev. A 79, 053620 (2009). 


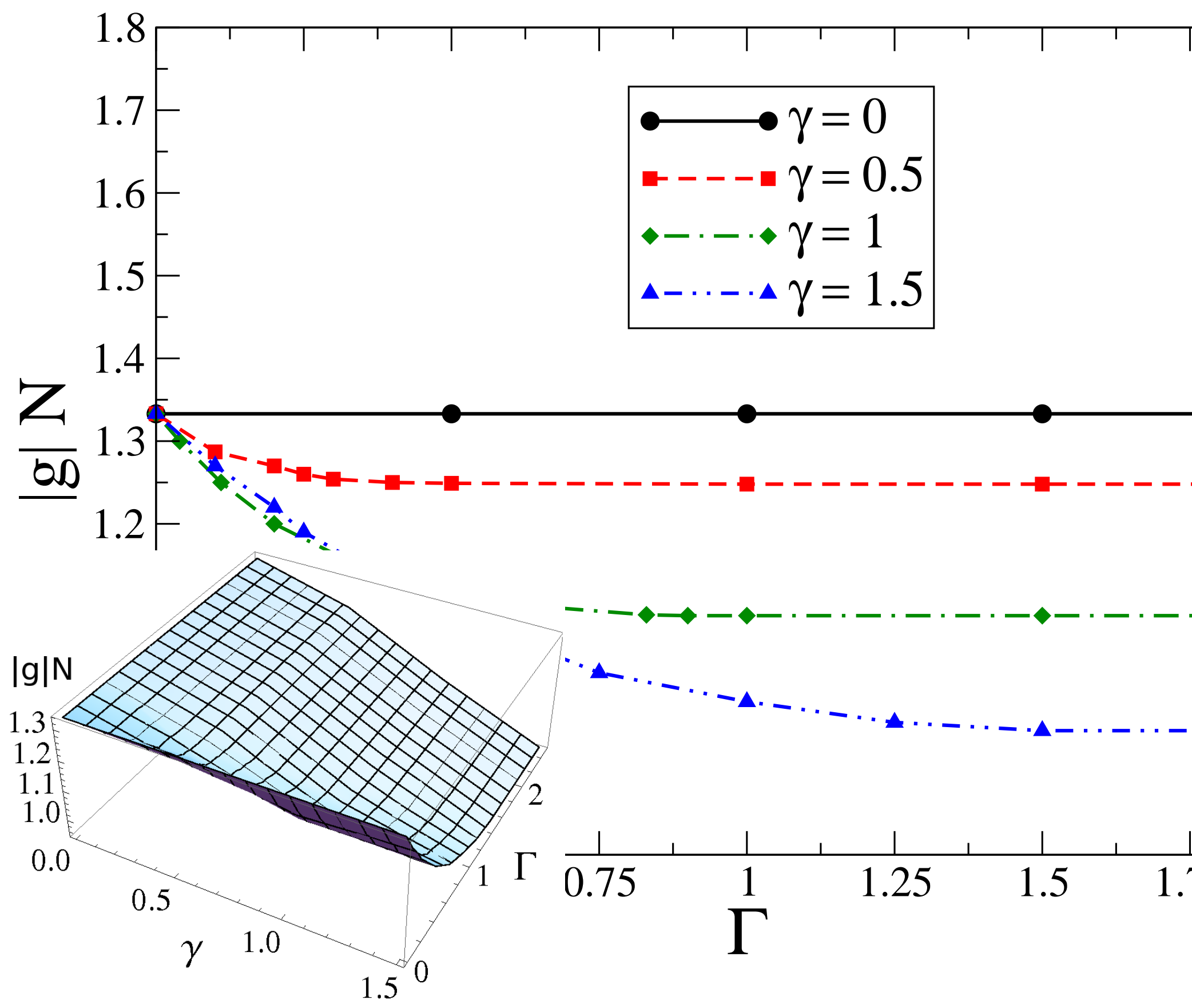

